\title{
Diagnosis and Treatment Algorythm in Lyme Neuroborreliosis
}

\author{
Țilea Brîndușa ${ }^{1 *}$, Bălașa Rodica1, Fodor Andrea1 ${ }^{1}$, Țilea loan ${ }^{1}$ \\ 1 University of Medicine and Pharmacy, Tîrgu Mureș, Romania
}

Lyme neuroborreliosis is an infection of the nervous system caused by spirochetes of the Borrelia burgdorferi sensulato group. Neurological clinical manifestations usually present a steady evolution and are different in patients from Europe compared to those from America, possibly due to vector agents and different bacterial species. Various diagnostic markers were studied in consideration of a clear or possible diagnosis of the disease, because evolution and complications depend on early diagnosis and initiation of therapy. The isolation of the bacterium is difficult, microscopic examination and the bacterial dezoxiribonucleic acid amplification shows low sensitivity. However, the diagnosis of Lyme neuroborreliosis is mainly based on serological methods that have a satisfactory sensitivity and specificity. A correct diagnosis can be performed by strictly respecting clinical guidelines and protocols and carefully interpreting the serological tests. The presence of anti-borrelia burgdorferi antibodies in the cerebrospinal fluid with evidence of intrathecal antibody production is the gold standard diagnosis of Lyme neuroborreliosis. Early administration of antibiotic treatment (third generation cephalosporins, cyclins, aminopenicillins) can produce the remission of neurological symptoms, the eradication of spirochetes in acute phase of the disease, thus avoiding the development of the chronic disease.

Keywords: Lyme neuroborreliosis, case definition, diagnosis, treatment

Received: 21 September 2015 / Accepted: 28 October 2015

\section{Introduction}

Lyme neuroborreliosis (LNB) is a clinical manifestation of Lyme borreliosis (LB), which affects the central nervous system (CNS) and peripheral nervous system (PNS) caused by spirochetes of the genus Borrelia burgdorferi $\mathrm{Bb})$ sensulato complex and spread by the bite of ticks of the genus Ixodes. Nerve structures are affected by spirochetes both in acute and late phase of the disease. A distinction between early (acute) and late (chronic) phase of LNB can be made. The acute phase occurs in less than 6 months after contacting an infected arthropod and chronic LNB sets in more than 6 months or even years later after the initial infection $[1,2]$.

The clinical manifestations of LNB are various and different in patients from Europe compared to those from America, probably due to different vector agents and bacterial species of the two continents. According to Steere the frequency of neurological manifestations in case of LB is about $15-25 \%$ [3].

\section{Clinical manifestations}

1. Early Lyme neuroborreliosis (LNB) include neurological manifestations of $\mathrm{Bb}$ spirochete infection, which occur in less than 6 months after the initial infection. In the USA, LB is associated with neurological manifestations in approximately $20 \%$ of the cases, while the dermatological manifestation, erythema migrans (EM) currently appears in $30 \%$ of the cases. Even in the initial phase of EM, in case of disseminated infection, symptoms like headache, neck stiffness and rarely discrete signs of encephalopathy

* Correspondence to: Brîndușa Țilea

E-mail: brindusa.tilea@umftgm.ro can appear. Neurological symptoms usually begin during the summer months, 4-6 weeks (in extreme cases 1-16 weeks) after the tick bite. Over $90 \%$ of patients are comprised in the group of early - onset LNB $[1,4]$.

\section{Central Nervous System Disorders}

Acute borrelia meningitis is the most common manifestation of early LNB. It often occurs in the secondary stage of the disease in approximately $20 \%$ of untreated patients, being more common in children and adolescents; $16 \%$ from all cases of lymphocytic meningitis are represented by this. Acute borrelia meningitis is often associated with cranial neuritis (the most affected nerve is the facial nerve) and radiculoneuritis, resulting in Bannwarth syndrome or meningoradiculitis (MR) which is a typical manifestation of LNB [5-7].

Acute borrelia meningitis can also appear as isolated meningitis by dominating the clinical picture, thus LB should be considered a possible cause of acute lymphocytic meningitis [8].

Symptoms of meningitis are fever $\left(38^{\circ} \mathrm{C}\right)$, pain (myalgia, arthralgia, cutaneous hyperesthesia), intracranial hypertension syndrome, meningeal irritation (neck stiffness, positive Kerning sign). These symptoms are often nonspecific, of mild intensity; they may occur isolated or accompanied by cranial radiculo neuropathy, nervous system manifestations (Bannwarth syndrome) or associated with signs of brain injury (confusion, ataxic hemiparesis with cerebellar dysarthria, symptoms of parkinsonism, etc) $[9,10]$.

Cerebrospinal fluid (CSF) pressure is usually in normal range, with moderate pleocytosis from tens to hundreds of elements, lymphocytes (10-1000 cellular elements/ $\left.\mathrm{mm}^{3}\right)$ [4]. 
The CSF protein concentration is usually slightly higher with values above $100 \mathrm{mg} / \mathrm{dl}$, sometimes reaching 300-400 $\mathrm{mg} / \mathrm{dl}$. Glycorachia, in most cases, is in normal limits, but documented clinical cases of hypoglycorachia with steady evolution were also reported. In the absence of etiological treatment, CSF alterations may persist for several months and anti-Bb antibodies for a few years without any other signs of disease progression $[2,11]$.

Lyme encephalomyelitis is a uni- or multifocal inflammatory manifestation of the CNS. Encephalitis or myelitis are most reported in European and American studies with different incidence. Brain parenchyma abnormalities are found in $20-50 \%$ of North American patients with Lyme meningitis. CNS manifestations frequency is generally low. This condition is reported more often in Europe and almost always accompanied by CSF pleocytosis and cranial neuritis [12].

CNS damage may occur isolated, but in most cases may be associated with the meningeal and peripheral one, with clinical manifestations of acute meningoencephalitis, acute meningoencephalomyelitis, acute polyradiculoneuritis encephalomyelitis. Cerebromedullary manifestations are generally single phase, slowly progressive and the cerebral white matter is affected [13].

Manifestations of Lyme encephalomyelitis are usually described with mild intensity and lack of severity such as: isolated Babinski sign, transient disorders of the sphincters, sleep and memory disorders and behavioural changes. In severe cases, brain damage can produce seizures, mental confusion, agitation, hallucinatory delirium, chorea, hemiparesis, cerebellar ataxia, choreoathetosis, dystonia [14].

Sometimes radicular inflammation intensifies, which can cause mild or moderate symptoms of myelitis, in most cases with a clinical picture of encephalomyelitis or pure myelitis, which can simulate multiple sclerosis (MS) or spinal cord compression [15].

\section{Peripheral Nervous System Disorders}

Disorders of the cranial nerve. Frequency of cranial nerve injuries is estimated to be $30-40 \%$ of the cases. The most common condition is uni- or bilateral facial nerve palsy, which often presents favourable evolution, recovery can be accomplished even without therapy in 1-2 months. Recovery rarely requires several months and in $10 \%$ of the cases it may be incomplete. Cranial nerve disorder in case of LB is more common in the secondary stage of the disease [16].

Other cranial nerves can also be involved especially the oculomotor and the trigeminal nerve, disorders of the $8^{\text {th }}$, $9^{\text {th }}, 12^{\text {th }}$ cranial nerve pairs can also be possible [3].

Optic neuritis is a condition that can occur isolated similar to facial nerve paralysis and should not be confused with idiopathic optic neuritis or MS.

When optic neuritis is the consequence of infection with $\mathrm{Bb}$ this may be accompanied by pleocytosis in the CSF and intrathecal synthesis of anti-Bb antibodies [17].
Radiculoneuritis occurs in approximately $1 / 3$ of patients suffering from neurological disorders, accompanied by pleocytosis in CSF (85\% of cases) and cranial neuropathy (70\% of cases). Symptomatology comprises radicular pain, paresthesia and/or hyperaesthesia, which usually appear on average 30 days after the initial infection; typical regions of appearance are cephalic extremity, the lower and upper extremities [4].

Intensity and metameric topography of pain and palsy are considered to be similar to the pain syndrome experienced in shingles $[18,19]$.

Pain syndrome can start as acute or subacute in character, usually at the level of the body or proximal part of the limbs and can reach full intensity within hours to several days. Once installed, pain can persist for weeks (6 weeks to 3-6 months) before spontaneous remission. Usually the pain syndrome has moderate intensity during the day, but it becomes exacerbated during the night; pain generally remains localized at the level of a dermatome or adjacent territory, rarely becoming diffuse or pluriradicular. Although not specific and often responsible for insomnia, the nocturnal appearance of pain syndrome has major clinical significance. Pain caused by radiculoneuritis is the first and in $20-25 \%$ of patients the only one neurologic symptom of early LNB [20].

Physical examination may be normal or reveal: hypoesthesia, muscle weakness and/or abolition of reflexes in the painful area or diffuse dysfunction. Radicular lesions may be accompanied or followed by unsteady paralytic peripheral or cranial nerve injury after a symptom-free interval of several weeks [21].

Etiological treatment, in comparison with the ineffectiveness of symptomatic therapy, has a beneficial effect on painful manifestations of the radicles. This favourable evolution of the post-antibacterial therapy, which generally occurs within 72 hours after onset, is an excellent argument in favour of the diagnosis of radiculoneuritis associated with LB $[4,22]$.

Peripheral motor nerve damage. Motor nerves damage, which is less common ( $20 \%$ of cases) than sensory nerves damage, is responsible for asymmetric paralysis. It is more often localized proximal than distal in some muscle groups, which are responsible for amyotrophy. Physical examination reveals diminished or abolished deep tendon reflexes in the area where paralysis or sometimes diffuse muscle damage occurs. The evolution of the disease can be prolonged and sequelae may appear. This type of paralysis often regresses in six to eight weeks $[6,23]$.

2. Late Lyme neuroborreliosis. Infection with $\mathrm{Bb}$ is considered to be one of the causes of late neurological disorders, which is supported by the following arguments: classic history of $\mathrm{LB}$, the presence of chronic lymphocytic meningitis, presence of specific anti-Bb antibodies in the CSF, regression during antibacterial therapy. Late neurological complications are cerebral, spinal or cerebromedullary [24]. 
The occurrence of late neurological manifestations after inoculation can vary in time. According to Logigian this period varies from 6 months to 17 years, on average 5 years [23].

Alterations in case of late LNB may be preceded by peripheral or central nervous manifestations of the early phase or on the contrary, which may be the first neurological complications of the disease $[11,13]$.

\section{Central Nervous System Disorders}

Chronic lymphocytic meningitisis a relatively rare manifestation of the CNS in case of late LNB, which is characterized by asymptomatic evolution, usually discovered accidentally and most often associated with late onset encephalitis [4].

Similar to lymphocytic meningitis, there are alterations in the CSF characterized by pleocytosis of several tens to several hundreds of elements $\left(80-400\right.$ cells $\left./ \mathrm{mm}^{3}\right)$ with a modified biochemistry, increased values of CSF proteins $100-300 \mathrm{mg} \%$ and occasionally cytoalbuminologic dissociation and normal or slightly decreased glycorrhachia may be observed. Generally high levels of immunoglobulins in the CSF are expressed; at this stage oligoclonal bands of immunoglobulin G (OCBs of $\mathrm{IgG}$ ) are present and the intrathecal synthesis of anti-Bb antibodies is also evident [25].

Progressive borrelia encephalomyelitisis the best defined late LNB with neurological complications. Most cases of progressive borrelia encephalomyelitis havebeen reported in Europe, but there are well documented cases in the US as well [16].

Both genders are equally affected, the average age of patients is 45 years, children are less affected than adults. There are several associated clinical aspects which occur gradually and with outbreaks, and sometimes undergo spontaneous regression. These manifestations can occur as disorders of higher cortical function of varying severity: impairment of memory and judgment, disturbances of attention, changes in character, apraxia, hallucinations, and dementia. Variations in alertness, mental confusion and even coma were also reported. In some patients may appear repetitive bouts of intense psychasthenia. Some authors reported focal disturbances (hemiparesis, hemianopia, cerebellar ataxia, parkinsonism), seizures and facial nerve palsy (mainly unilateral). The clinical picture and outbreaks of progressive borrelia encephalomyelitis can simulate MS or stroke [26,27].

Spinal cord is affected in $50 \%$ of the cases, mainly under the form of progressive spastic paraparesis or quadriparesis followed by bladder dysfunction. The involvement of $\mathrm{Bb}$ in late neurological manifestations is difficult to prove, the isolation of spirochete in the CSF is remarkable, but the most reliable diagnostic argument is the emphasized intrathecal synthesis of anti-Bb antibodies [23].

Lyme encephalopathy (LE) occurs as a neuropsychiatric disorder several months or years after the time of infection with $\mathrm{Bb}$, and is classified as late stage LB [11]. It is a cognitive disorder of moderate severity that mostly affects memory and learning, occasionally followed by psychiatric symptoms or drowsiness, which cannot be associated with neurological pathology signs or any modifications revealed by medical imaging [28].

\section{Peripheral Nervous System Disorders}

Polyneuropathy and neuropathy associated with sclerotic lesions. Many patients with late LB present a mild form of multifocal polyneuropathy which is different from earlyonset meningeal polyneuritis, because it does not improve spontaneously. The most common symptoms are limb paraesthesia, which are often intermittent, asymmetric, focal, affecting both the lower and upper limbs; rarely, radicular pain can also be present. A quarter of these patients presented carpal tunnel syndrome too. Distal paraesthesia generally occurs in the median nerve distribution and it is exacerbated by night-time or the use of hands; in $2 / 3$ of the cases this condition is bilateral. The presence of physical signs in case of neuropathy are rare [29].

Symptoms are accompanied by objective changes - suppressed deep tendon reflexes and/or atrophies; these symptoms are usually asymmetric and more intense in the affected skin area .

Late LNB must be considered when chronic lymphocytic meningitis or meningoencephalitis is diagnosed, especially if they are associated with peripheral cranial neuropathy or radiculoneuropathy $[3,4]$.

It is difficult to confirm the diagnosis because the demonstration of anti- $\mathrm{Bb}$ antibodies presence in the patient's serum is not a sufficient argument. On the other hand, the intrathecal synthesis of anti- $\mathrm{Bb}$ antibodies appears in 90\% of early-onset LNB cases and occurs frequently in patients with chronic encephalomyelitis, but it is rare in patients with late-onset encephalopathy and in patients with peripheral neuropathy [30].

Sometimes response to early antibiotic treatment may be a useful argument to confirm the diagnosis of late LNB, although it is a known fact that similar to tertiary syphilis, there are cases that do not respond to therapy [31].

\section{Diagnosis of Lyme neuroborreliosis}

Lyme neuroborreliosis is the clinical manifestation of an infectious disease with a multisystemic character which always needs to be correlated with the common trigger of the underlying disease: the infection of the whole body with Bb. spirochete.

Actually an accurate LB diagnosis continues to be a problematic issue; this happens until a "gold standard" diagnostic test will be developed.Rigorous criteria are required to establish the cause-effect association between a given neurological syndrome and infection with $\mathrm{Bb}[6]$.

Currently the diagnosis of LNB is based on serological methods. The existing serological tests are not fully satisfactory, both in terms of sensitivity (due to slow humoral response) as well as specificity (several false positive results) 
and the lack of ability to define early-onset from late-onset infection due to the persistence of antibodies in both treated and untreated forms.

It is difficultto establish a clearly positive LNBdiagnosis, which is usuallyset up basedon the clinical neurologicalmanifestations andserological investigationsboth in CSFandserum [32].

\section{Laboratory tests}

CSF analysis. In Europe LNB is associated with CSF pleocytosis with cell count ranging from $10-1000$ cells $/ \mathrm{mm}^{3}$, where most of them are lymphocytes with increased values of CSF proteins $100-300 \mathrm{mg} / \mathrm{dl}$ and a normal glycorrhachia and oligoclonal $\operatorname{IgG}$ bands in about $90 \%$ of the cases [1].

Serological tests. Specific anti-Bb antibodies (IgM, IgG) in serum and CSF can be detected by ELISA test. Most laboratories use a two-step approach, a positive ELISA is always followed by a Western blot test. As a confirmative test, Western blot has a specificity of $95 \%$.

The diagnostic sensitivity of ELISA screening test in case of early-onset LNB is 70-90\% and in late-onset LNB is 90$100 \%$. The specificity of the diagnostic tests for serum antibodies is low because seropositivity in general population is between $5-20 \%$. IgM anti-Bb antibodies may be false positive because of oligoclonal stimulation and $\operatorname{IgM}, \operatorname{IgG}$ antibodies can persist a long period after a complete treatment. In order to demonstrate the intrathecal production of specific anti- $\mathrm{Bb}$ antibodies calculation methods are used that take into account the blood-brain barrier dysfunction - antibody index (AI).

The intrathecal production of IgM antibodies presents an increased sensitivity in short term LNB. A positive AI may persist years after treatment. AI sensitivity is about $80 \%$ in short-term LNB and 100\% for long-term LNB. Diagnostic specificity in a study was $63 \%[2,6,9]$.

Microscopic examination - Bb can be identified in cultures, but at a higher density of $10^{5}$ germ cells by using darkfield microscopy, but the results are negative for blood or CSF testing. Immunostaining based on specific anti- $\mathrm{Bb}$ antibody index is a widely used technique to detect the presence of $\mathrm{Bb}$ in ticks. Modified Dieterle or Steiner stain of the silver plating kit produces a golden yellow background that contrasts with the diversely coloured spirochetes, brown-blue-black. De Vago staining uses dark-field microscopy to view spirochetes [18].

The most used immunohistochemical technique is immunogold-silver staining coupled with double epi-illumination: an immunofluorescence illumination emphasizing spirochetes covered by silver particles that reflects light on a dark background and an optical microscope illumination, visualizing histological structures emphasized by hematoxylin-eosin staining. The method is extremely laborious and it is more frequently used in research laboratories. This method is not recommended for routine LNB diagnosis $[17,33]$.
Polymerase chain reaction (PCR) technique - The diagnostic sensitivity of PCR in late-onset LNB is extremely low; according to some studies, in CSF for early-onset LNB is between $10-30 \%$.

Due to low sensitivity and unknown specificity, PCR is not recommended as a diagnostic tool in patients with chronic symptoms or as a method for monitoring treatment [34].

Direct hybridization for $\mathrm{Bb}$ detection can be used only when the number of spirochetes in the examined sample is of thousands (rare possibility); therefore techniques that perform DNA amplification are needed.

The classic risk is represented by false-positive test results while amplifying the unwanted copies of foreign DNA through contamination, false-negative test results are also possible. These cases are due to either a DNA quantity below the detection limit of the used method (generally less than 10 bacteria) or for the presence of polymerase inhibitors [35].

False-negative results can occur if one of the primers is not properly hybridized with the 'target' bacterial DNA. This situation is possible in case of Borreliaburgdorferi - a bacterium presenting high genomic heterogeneity especially in isolated strains in Europe [36].

Culture - spirochete $\mathrm{Bb}$ can be sampled from the CSF, skin and blood by using the modified Barbour-StoennerKelly culture at $30-40^{\circ} \mathrm{C}$ but the method is laborious, time consuming; the incubation period is up to 12 weeks due to slow growth of spirochete in vitro. Microscopic detection of $\mathrm{Bb}$ can produce false-positive test results. After isolating the spirochete this should be identified either by using a PCR or by specific monoclonal antibodies marking. Sensitivity is $10-30 \%$ for CSF in case of early-onset LNB and 50-70\% for skin biopsies [13].

Chemokine CXCL13- recent studies have demonstrated that chemokine CXCL13, which attracts B cells, shows high values in the CSF of patients with early-onset LNB. In a class II study the diagnostic sensitivity of CXCL13ELISA was $100 \%$ in early-onset LNB and the specificity was $63 \%$ reaching normal values in over $70 \%$ of the patients after treatment [37].

The test is recommended for seronegative patients during the early phase and for therapy assessment $[38,39]$.

The study of $T$ cell proliferation. A cell-mediated immunity test is used for the identification of exposure to $\mathrm{Bb}$ because the earliest immune response after the onset of the infection is the T-cell response. Cell-mediated immunity response is best stimulated by complete cellular Bbantigens in comparison to soluble antigens from isolated spirochetes. This response is emphasized in the CSF and synovial fluid mononuclear cells. In case of chronic infection with $\mathrm{Bb}$ a significant decrease in CD57 lymphocytes was observed. The decrease of CD57 cell degree is assessed as an indicator of disease activity. Cell-mediated immunity technique is less sensitive than serological methods, as well as B-cell response mediated by T-cell response and after completing an effective treatment [40]. 
To confirm LNB diagnosis is mandatory to document the presence of anti $\mathrm{Bb}$ antibodies (IgM and $\mathrm{IgG}$ ) from CSF and serum, the antibody index using ELISA and Western blot techniques and CSF inflammatory signs.

Culture and PCR are recommended only in early stages of LNB. Today other diagnostic tools are not recommended.

The European Federation of Neurological Societies (EFNS) recommends the following criteria in order to establish possible and defined LNB diagnosis [2].

Defined $L N B$ - requires the following three criteria:

- Suggestive neurological symptoms of LNB - with the exclusion of other causes

- CSF pleocytosis

- Specific anti-Bb antibodies in the CSF

Possible $L N B$ - requires the presence of two out of the three criteria mentioned above. If the third criterion is missing after a period of 6 weeks the detection of IgG anti$\mathrm{Bb}$ antibodies in the CSF is required.

The National Centre for the Surveillance and Control of Communicable Diseases in Romania recommends the use of a serologic diagnostic algorithm for early-onset and late-onset LNB. In order to confirm LNB, IgM or IgG anti-Bb antibodies must be present in both the serum and CSF, sampled at the same time by using the Western-blot technique [41]. (Figure 1)

\section{Treatment of Lyme neuroborreliosis}

The treatment of early LNB compared to other determinations requires special therapeutic features. The aim of the antibacterial therapy is twofold: the elimination of subjec- tive complaints and eradication of spirochetes in order to prevent the infection to become chronic.

In recent years, therapeutic modalities of LNB were updated taking into account in vitro activity of antibiotics on Borrelia burgdorferi, pharmacokinetics, intrathecal diffusion, mode of administration, individual tolerance [7].

Antibiotics that achieve concentrations in the CSF and cerebral white matter recommended by the European Concerted Action on Lyme Borreliosis (EUCALB) for the treatment of early- and late-onset LNB are cephalosporins of third generation (Ceftriaxone, Cefotaxime), betalactams (Penicillin G), aminopenicillins (Amoxicillin) and cyclins (Doxycycline) [41,42]. (Table I)

Usually, route of administration is intravenous, except for Doxycycline which is administered orally and the length of therapy is 14 days in case of early-onset LNB and 30 days in late-onset LNB $[42,43]$. The use of antibiotics in combination with corticosteroids facilitates healing, but corticosteroidsdo not have noticeable effect on motor deficit. In randomized clinical trials no differences were observed among Penicillin G administered in high doses, Ceftriaxone, Cefotaxime and Doxycycline [44].

Ceftriaxone therapy at a dose of $2 \mathrm{~g} /$ day for a period of 14 days seems to be the primary treatment in case of early-onset LNB and $2 \mathrm{~g} /$ day for a period of 30 days in late LNB. Doxycycline is not indicated in children under 12 and pregnant women [45]. Length of therapy may be extended in early LNB up to 30 days if clinical symptoms do not remit or the inflammatory process in the CNS and PNS is stationary $[46,47]$.

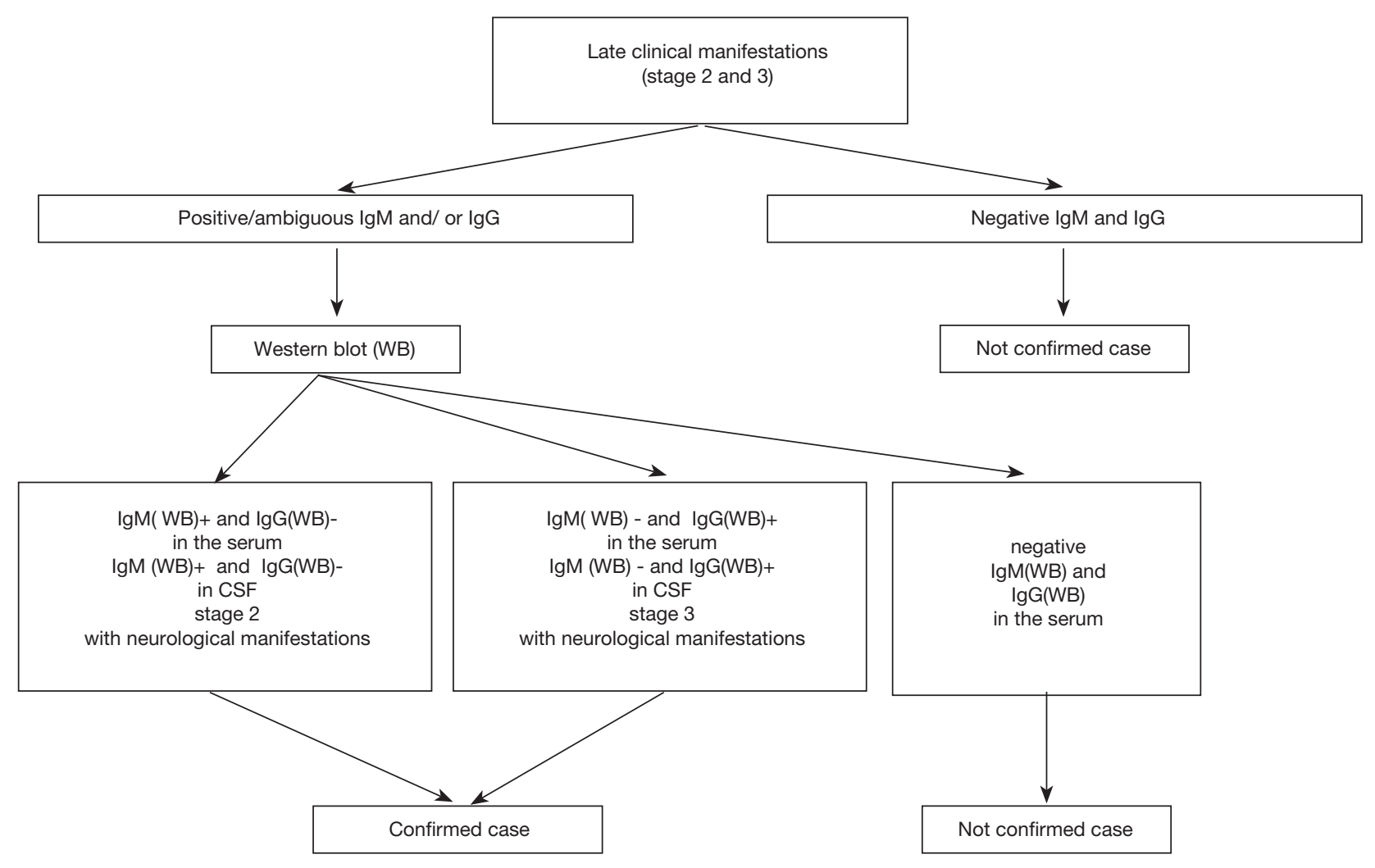

Fig. 1. Romanian algorithm for the serological diagnosis of LNB [adapted from 40] 
Table I. Antibiotics used in the treatment of LNB [adapted from 41]

\begin{tabular}{|c|c|c|c|c|c|}
\hline Clinical form of disease & Antibiotic & Dosage & Administration & Regularity (days) & $\begin{array}{c}\text { Duration } \\
\text { (days) }\end{array}$ \\
\hline \multirow{5}{*}{ Early-onset LNB } & Ceftriaxone & $2 \mathrm{~g}$ & i.v. & 1 & 14 \\
\hline & Cefotaxime & $2 \mathrm{~g}$ & i.v. & 2 & 14 \\
\hline & Penicillin G & $3 \mathrm{MUI}$ & i.v. & 3 & 14 \\
\hline & Doxycycline & $200 \mathrm{mg}$ & orally & 1 & 14 \\
\hline & Amoxicillin & $2 \mathrm{~g}$ & orally & 3 & 14 \\
\hline Late-onset LNB & $\begin{array}{l}\text { Identical with the treatment of early- } \\
\text { onset LNB, but with a duration of } \\
30 \text { days }\end{array}$ & & & & 30 \\
\hline
\end{tabular}

\section{Conclusion}

The neuroborreliosis safety diagnosis and treatment are performed by respecting the case definition and medical protocols recommended by European and national guidelines.

\section{Acknowledgments}

This paper is partially supported by Research Grant no.13894/2014, by the University of Medicine and Pharmacy of Tirgu Mures, Romania and SC Centrul Medical Topmed SRL, Tirgu Mures, Romania.

\section{Conflict of interests}

The authors declare that they have no conflict of interest

\section{References}

1. Stanek G, Fingerle V, Hunfeld KP, et al. Lyme borreliosis: clinical case definitions for diagnosis and management in Europe. ClinMicrobiollnfect. 2011;17:69-79.

2. Mygland $A$, Ljostad $U$, Fingerle $V$, et al. EFNS guidelines on the diagnosis and management of European Lyme neuroborreliosis. Eur $\mathrm{J}$ Neurol.2010;17:8-16.

3. Steere AC. Lyme disease. N Engl J Med. 2001;345:115-25.

4. Halperin JJ. Neuroborreliosis. Am J Med. 1995;98(4):52-6.

5. Kruger H, Reuss K, Pulz M, et al. Meningoradiculitis and encephalomyelitis due to Borreliaburgdorferi: a follow-up study of 72 patients over 27 years. J Neurol. 1989;236:322-8.

6. Halperin JJ, Shapiro ED, Logigian E, et al. Practice parameter: treatment of nervous system Lyme disease (an evidence-based review): report of the Quality Standards Subcommittee of the American Academy of Neurology. Neurology.2007;69:91-102.

7. Wormser GP, Dattwyler RJ, Shapiro ED, et al. The clinical assessment, treatment, and prevention of Lyme disease, human granulocytic anaplasmosis, and babesiosis: clinical practice guidelines by the Infectious Diseases Society of America. Clin Infect Dis. 2006;43(9):1089134.

8. Hansen K, Lebech AM. The clinical and epidemiological profile of Lyme neuroborreliosis in Denmark 1985-1990.A prospective study of 187 patients with Borreliaburgdorferi-specific intrathecal antibody production. Brain. 1992;115(Pt2):399-423.

9. Ljostad U, Skarpaas T, Mygland A. Clinical usefulness of intrathecal antibody testing in acute Lyme neuroborreliosis. Eur J Neurol.2007;14(8):873-6.

10. Hammers-Berggren S, Hansen K, Lebech AM, Karlsson M. Borreliaburgdorferi-specific intrathecal antibody production in neuroborreliosis: a follow-up study. Neurology.1993;43(1):169-75.

11. Halperin JJ, Volkman DJ, Wu P. Central nervous system abnormalities in Lyme neuroborreliosis. Neurology. 1991;41(10):1571-82

12. OschmannP, Dorndorf W, Hornig C, et al. Stages and syndromes of neuroborreliosis. J Neurol. 1998;245:262-72.

13. O'Connell S. Lyme borreliosis: current issues in diagnosis and management. CurrOpin Infect Dis. 2010;23(3):231-5.

14. Skogman $B H$, Croner $S$, Nordwall $M$, et al. Lyme neuroborreliosis in children : a prospective study of clinical features, prognosis, and outcome.Pediatr Infect Dis J.2008;27(12):1089-94.
15. SkeieGO, Eldoen G, Skeie BS, et al.Opsoclonus myoclonus syndrome in two cases with neuroborreliosis. Eur J Neurol. 2007;14(12): el-2.

16. Wormser GP, Dattwyler RJ, Shapiro ED et al. The clinical assessment, treatment, and prevention of Lyme disease, human granulocytic anaplasmosis, and babesiosis: clinical practice guidelines by the Infectious Diseases Society of American. Clin Infect Dis. 2006;43(9):1089-1134.

17. Aguero-Rosenfeld ME, Wang G, Schwartz I, Wormser GP. Diagnosis of Lyme borreliosis. Clin Microbiol Rev. 2005;18(3):484-509.

18. Stanek G, Strle F. Lyme borreliosis- European perspective. Infect Dis Clin North Am. 2008;22(2):327-39.

19. Strle F, Stanek G. Clinical manifestations and diagnosis of Lyme borreliosis. Curr Probl Dermatol 2009;37:51-110.

20. Halperin J, Luft BJ, Volkman DJ, Dattwyler RJ. Lyme neuroborreliosis. Peripheral nervous system manifestations. Brain. 1990;113(Pt4):120721.

21. Hansen K, Lebech AM. Lyme neuroborreliosis: a new sensitive diagnostic assay for intrathecal synthesis of Borreliaburgdorferi-specific immunoglobulin G, A, and M. Ann Neurol. 1991;30(2):197-205.

22. Ljostad U,Skogvoll E, Eikeland R, et al. Oral doxycycline versus intravenous ceftriaxone for European Lyme neuroboreliosis: a multicenter, non-inferiority, double-blind, randomised trial. Lancet Neurol. 2008;7(8):690-95.

23. Kruger H, Reuss K, Pulz M, et al. Meningoradiculitis and encephalomyelitis due to Borreliaburgdorferi: a follow-up study of 72 patients over 27 years. J Neurol. 1989;236(6):322-28.

24. Logigian EL, Kaplan RF, Steere AC. Chronic neurologic manifestation of Lyme disease. N Engl J Med. 1990;323(21):1438-44.

25. Mygland A, Skarpaas T, Ljostad U. Chronic polyneuropathy and Lyme disease. Eur J Neurol. 2006;13(11):1213-15.

26. Topakian R, Stieglbauer K, Nussbaumer K, Aichner FT.Cerebral vasculitis and stroke in lyme neuroborreliosis. Two case report and review of current knowledge. Cerebrovasc Dis. 2008;26(5):455- 61.

27. Charles V, Duprez TP, Kabamba B, et al. Poliomyelitis-like syndrome with matching magnetic resonance features in a case of Lyme neuroborreliosis. J NeurolNeurosurg Psychiatry. 2007;78:1160-61.

28. Wilke M, Eiffert $H$, Christen HJ, Hanefeld F. Primarily chronic and cerebrovascular course of Lyme neuroborreliosis : case report and literature review. Arch Dis Child 2000;83(1):67-71.

29. Kindstrand E, Nilsson BY, Hovmark A et al. Polyneuropathy in late Lyme neuroborreliosis - a clinical, neurophysiological and morphological description. ActaNeurol Scand. 2000;101:47-52.

30. Hehir MK 2nd, Logigian EL. Infectious neuropaties. Continuum (Minneap Minn). 2014;20(5 Peripheral Nervous System Disorders):1274-92.

31. Mygland A, Skarpaas T, Ljostad U. Chronic polyneuropathy and Lyme disease. Eur J Neurol. 2006;13:1213-15.

32. Wilske B, Fingerle V, Schulte-Spechtel U. Microbiological and serological diagnosis of Lyme borreliosis. FEMS Immunol Med Microbiol. 2007;49:1321.

33. Tilea B,Tripon G, Voidazan S, Tilea I. Serological, clinical and epidemiological aspects of Lyme borreliosis in Mures County, Romania. Rev Romana Med Lab. 2014;22(2):233-34.

34. LebechAM, Hansen K, Brandrup F, et al. Diagnostic value of PCR for detection of Borreliaburgdorferi DNA in clinical specimens from patients with erythema migrans and Lyme neuroborreliosis. MolDiagn. 2000;5:139-50

35. Ornstein K, Berglund J, Bergstrom S, et al. Three major LymeBorreliagenospecies (Borreliaburgdorferisensustricto, B. afzelii and B. garinii) identified by PCR in cerebrospinal fluid from patients with neuroborreliosis in Sweden .Scand J Inf Dis. 2002;34(5):341-46.

36. Hashimoto $\mathrm{Y}$, Takahashi $\mathrm{H}$, Kishiyama $\mathrm{K}$, et al. Lyme disease with facial nerve palsy: rapid diagnosis using a nested polymerase chain reaction- 
restriction fragment length polymorphism analysis. $\mathrm{Br} J$ Dermatol. 1998;138(2):304-9

37. Ljostad U, Mygland A. CSF B- lymphocyte chemoattractant (CXCL13) in the early diagnosis of acute Lyme neuroborreliosis. J Neurol. 2008;255:732-37.

38. Rupprecht TA, Pfister HW, Angele B, et al. The chemokine CXCL13 (BLC): a putative diagnostic marker for neuroborreliosis. Neurol. 2005;65(3):448-50.

39. Rupprecht TA, Kirschning CJ, Popp B, et al.Borreliagarinii induces CXCL13 production in human monocytes through Toll-like receptor 2. Infect Immun. 2007;75(5):4351-56.

40. Stricker RB, Winger EE. Decreased CD57 lymphocyte subset in patients with chronic Lyme disease.ImmunolLett. 2001;76(1):43-48.

41. Centrul national de Supravegheresi Control al BolilorTransmisibile-Boala Lyme metodologie de supraveghere: Lyme borreliosis available at http:// www.cnscbt.ro/index.php/metodologii/boala-lyme/10-boala-lyme- metodologie-de-supraveghere-2014/file

42. European Concerted Action on Lyme borreliosis-Diagnosis, treatment: Case Definition EUCALB available at http://eucalb.com

43. Karkkonen K, Stiernstedt SH, Karlsson M. Follow-up of patients treated with oral doxycycline for Lyme neuroborreliosis. Scand J Infect Dis. 2001;33(4):259-62.

44. Fallon BA, Keilp JG, Corbera KM, et al. A randomized, placebo-controlled trial of repeated IV antibiotic therapy for Lyme encephalopathy. Neurol. 2008;70(13):992-1003.

45. Ayaslioglu E, Erkek E, Oba AA, CebeciogluE. Doxycycline- induced staining of permanent adult dentition. Aust Dent J. 2005;50:273-75.

46. Fallon BA, Levin ES, Schweitzer PJ, Hardesty D. Inflammation and central nervous system Lyme disease. Neurobiol Dis. 2010;37(3):534-41.

47. Ljostat U, Skogvoll E, Eikeland R, et al. Oral doxycycline versus ceftriaxone for European Lyme neuroborreliosis: a multicentric, noninferiority, double blind, randomised trial. Lancet Neurol 2008; 7(8):690-5. 